\title{
Chiral Symmetry Breaking in the Nambu-Jona-Lasinio Model
}

\section{in External Constant Electromagnetic Field}

\author{
A.Yu. Babansky ${ }^{1}$, E.V. Gorbar ${ }^{2}$, and G.V. Shchepanyuk ${ }^{3}$ \\ ${ }^{1}$ Kiev State University, Ukraine \\ 2 Bogolyubov Institute for Theoretical Physics, Ukraine \\ ${ }^{3}$ Institute of Mathematics, Ukraine
}

\begin{abstract}
Dynamical chiral symmetry breaking $(\mathrm{D} \chi \mathrm{SB})$ is studied in the NambuJona-Lasinio model for an arbitrary combination of external constant electric and magnetic fields. In $3+1$ dimensions it is shown that the critical coupling constant increases with increasing of the value of the second invariant of electromagentic field $\vec{E} \cdot \vec{B}$, i.e. the second invariant inhibits $D \chi S B$. The case of $2+1$ dimensions is simpler because there is only one Lorentz invariant of electromagnetic field and any combination of constant fields can be reduced to cases either purely magnetic or purely electric field.
\end{abstract}


In works [1-3] it was first shown in the so called ladder approximation that quantum electrodynamics (QED) in the regime of strong coupling has a new phase with dynamically broken chiral symmetry. The new phase of QED possesses very interesting properties from the theoretical viewpoint [4-6]. There were attempts to use them for modelling electroweak symmetry breaking in technicolor-like models [7]. However, at present the new phase of QED has little relevance to experiment because we need a strong coupling constant $\alpha_{c} \approx 1$ (recall that the physical value of electromagnetic coupling constant is $\left.\alpha_{c}=\frac{1}{137} \ll 1\right)$.

However, as was suggested in [8, 9], the situation may drastically change in the presence of strong external electromagnetic fields where dynamical chiral symmetry breaking $(D \chi S B)$ may occur at the regime of weak coupling. A breakthrough in this direction was made in $[10,11]$, where, in the framework of the Nambu-Jona-Lasinio (NJL) model [12], it was shown that $D \chi S B$ takes place in an external constant magnetic field at any small attraction between fermions both in $2+1$ and $3+1$ dimensions (note that the fact that external magnetic field enhances $D \chi S B$ was first noted in the NJL model in [13] (see also [14])).

As shown in $[10,11]$, in the infrared, the dynamics of fermions in magnetic field in $2+1$ and $3+1$ dimensions resembles the dynamics of fermions in $0+1$ and $1+1$ dimensions, respectively. Therefore, we have an effective reduction of dimension of space-time by 2 units and as a result the critical value of the coupling coustant in external magnetic field is equal to zero. It was latter shown in [15] that the same effect takes place in QED in external magnetic field. Note that although the critical value of coupling constant is zero extremely strong magnetic fields $\left(|\vec{B}| \geq 10^{13} G\right)$ are necessary for experimentally significant consequences because the correction to the physical mass of electron is very tiny for weak magnetic fields.

The case of constant electric field was considered in [13] where it was shown that the value of the critical coupling constant is more in this case than in the case without electric field. In the present work we study $\mathrm{D} \chi \mathrm{SB}$ in the NJL model in the 
case of an arbitrary combination of constant electric and magnetic fields in $3+1$ and $2+1$ dimensions. We first consider the case of $3+1$ dimensions. As well known, electromagnetic field has two Lorentz invariants $f_{1}=\frac{1}{2} F_{\mu \nu} F^{\mu \nu}=\vec{B}^{2}-\vec{E}^{2}$ and $f_{2}=\frac{1}{2} \varepsilon^{\mu \nu \alpha \beta} F_{\mu \nu} F_{\alpha \beta}=\vec{E} \cdot \vec{B}$. Since $\mathrm{D} \chi \mathrm{SB}$ was already studied in cses of purely electric and magnetic constant external fields when only the first invariant $f_{1}$ of electromagnetic field is not equal to zero, in the present work we consider $\mathrm{D} \chi \mathrm{SB}$ in the case where $f_{2} \neq 0$.

The Lagrangian of the NJL model [12] in an external electromagnetic field reads

$$
\mathcal{L}=\sum_{j=1}^{N} i \bar{\Psi}_{j} \gamma^{\mu} D_{\mu} \Psi_{j}+\frac{G}{2} \sum_{j=1}^{N}\left[\left(\bar{\Psi}_{j} \Psi_{j}\right)^{2}+\left(\bar{\Psi}_{j} i \gamma_{5} \Psi_{j}\right)^{2}\right]
$$

where $D_{\mu}$ is the covariant derivative $D_{\mu}=\partial_{\mu}+i e A_{\mu}$ and $j=1,2, \ldots, N$ flavor index. Lagrangian (1) is invariant with respect to the $U_{L}(N) \times U_{R}(N)$ chiral group. By using auxiliary fields $\pi$ and $\sigma$, we can rewrite (1) in the following form:

$$
\mathcal{L}=\sum_{j=1}^{N}\left[i \bar{\Psi}_{j} \gamma^{\mu} D_{\mu} \Psi_{j}-\bar{\Psi}_{j}\left(\sigma_{j}+i \gamma_{5} \pi_{j}\right) \Psi_{j}-\frac{1}{2 G}\left(\sigma_{j}^{2}+\pi_{j}^{2}\right)\right]
$$

By taking integrals over fermion fields, we obtain the effective action for $\pi$ and $\sigma$ fields

$$
\Gamma(\sigma, \pi)=-i \sum_{j=1}^{N} \operatorname{Tr} \operatorname{Ln}\left[i \gamma^{\mu} D_{\mu}-\left(\sigma_{j}+i \gamma_{5} \pi_{j}\right)\right]-\frac{1}{2 G} \int d^{4} x\left(\sigma_{j}^{2}+\pi_{j}^{2}\right) .
$$

To obtain the effective potential for $\sigma$ and $\pi$ fields, it suffices to consider the case of constant fields $\sigma=$ const, $\pi=$ const. Since the effective action is invariant with respect to the $U_{L}(N) \times U_{R}(N)$ chiral symmetry, the effective potential depends on $\pi$ and $\sigma$ fields only through the chirally invariant combination $\rho^{2}=\sum_{j=1}^{N}\left(\sigma_{j}^{2}+\pi_{j}^{2}\right)$. Therefore, in what follows it is sufficient to set $\pi_{k}=0, \sigma_{k}=0$ for $k=2, \ldots, N$ and consider the effective potential only for the field $\sigma_{1}$ which we simply denote $\sigma$. Thus,

$$
\Gamma(\sigma)=-i \operatorname{Tr} \operatorname{Ln}\left[i \gamma^{\mu} D_{\mu}-\sigma\right]-\frac{1}{2 G} \int d^{4} x \sigma^{2} .
$$

By using the method of proper time [16, 17], we represent the first term in (4) as 
follows:

$$
-i \operatorname{Tr} \operatorname{Ln}\left(i D_{\mu} \gamma^{\mu}-\sigma\right)=-\frac{i}{2} \operatorname{Tr} \operatorname{Ln}\left(D^{2}+\sigma^{2}\right)=\int \frac{i}{2 s} \operatorname{tr}\left\langle x\left|e^{-i s\left(D^{2}+\sigma^{2}\right)}\right| x\right\rangle d s d^{4} x
$$

As well known [17], vacuum of QED is not stable in an external electric field and the effective potential has an imaginary part which defines the rate of birth of fermion-antifermion pairs from vacuum per unit volume. Since we study the problem of $D \chi S B$, we can ignore this effect and consider only the real part of effective potential which is equal to

$$
V(\sigma)=\frac{\sigma^{2}}{2 G}+\frac{N}{8 \pi^{2}} v \cdot p . \int_{1 / \Lambda^{2}}^{\infty} d s \frac{1}{s} e^{-s \sigma^{2}} M \operatorname{coth}(M s) L \cot (L s),
$$

where $L^{2}=e^{2} \frac{\sqrt{f_{1}^{2}+4 f_{2}^{2}}-f_{1}}{2}, M^{2}=e^{2} \frac{\sqrt{f_{1}^{2}+4 f_{2}^{2}}+f_{1}}{2}$, and $f_{1}=\vec{B}^{2}-\vec{E}^{2}$ and $f_{2}=\vec{E} \cdot \vec{B}$ are two invariants of electromagnetic field. In (6) we introduced a cut-off $\frac{1}{\Lambda^{2}}$ and v.p. of the integral in $\mathrm{s}$ is present because we consider only the real part of the effective potential (recall that the imaginary part of the effective potential is given by residues in poles of $\cot (L s))$. The gap equation $\delta V /\left.\delta \sigma\right|_{\sigma=m}=0$ has the form

$$
\frac{1}{G}-\frac{N}{4 \pi^{2}} v \cdot p \cdot \int_{1 / \Lambda^{2}}^{\infty} d s e^{-s m^{2}} M \operatorname{coth}(M s) L \cot (L s)=0 .
$$

This gap equation was investigated in cases where only the first invariant of electromagnetic field is not equal to zero, i.e. for cases of purely electric and magnetic external fields. In this paper we study how the presence of nonzero electric field parallel to magnetic field $(\vec{E} \cdot \vec{B} \neq 0)$ affects $D \chi S B$. By using some inequalities, we first analytically obtain an estimate from below for the critical coupling constant. We add and subtract $1 / \mathrm{s}$ to $M \operatorname{coth}(M s)$ in the gap equation (7). Then

$$
\begin{array}{r}
\text { v.p. } \int_{1 / \Lambda^{2}}^{\infty} d s e^{-s m^{2}} M \operatorname{coth}(M s) L \cot (L s)=\Lambda^{2}-\frac{\pi}{2} L+ \\
v . p . \int_{1 / \Lambda^{2}}^{\infty} d s e^{-s m^{2}}(M \operatorname{coth}(M s)-1 / s) L \cot (L s),
\end{array}
$$

where we used the result [13] v.p. $\int_{1 / \Lambda^{2}}^{\infty} d s e^{-s m^{2}} L \frac{\cot (L s)}{s}=\Lambda^{2}-\frac{\pi}{2} L$. Further, we represent the integral in (8) as a sum of two integrals $\int_{1 / \Lambda^{2}}^{\infty}=\int_{1 / \Lambda^{2}}^{\frac{\pi}{2 L}}+\int_{\frac{\pi}{2 L}}^{\infty}$ (note that 
$\frac{\pi}{2 L}$ is the first zero of $\left.\cot (L s)\right)$. We now consider the integral from $\frac{\pi}{2 L}$ to infinity. Since $\operatorname{coth} \mathrm{x} \leq 1 / \mathrm{x}+1$ for $x>0$, we have

$$
v \cdot p \cdot \int_{\frac{\pi}{2 L}}^{\infty} d s e^{-s m^{2}}(M \operatorname{coth}(M s)-1 / s) L \cot (L s) \leq v \cdot p \cdot \int_{\frac{\pi}{2 L}}^{\infty} d s e^{-s m^{2}} M L \cot (L s)
$$

Integrating by part, we obtain

$$
\begin{array}{r}
\text { v.p. } \int_{\frac{\pi}{2 L}}^{\infty} d s e^{-s m^{2}} M L \cot (L s)=M \int_{\frac{\pi}{2 L}}^{\infty} e^{-m^{2} s} d(\ln (2|\sin L s|)) \\
=-M \ln \left(2\left|\sin \frac{\pi}{2}\right|\right)+m^{2} M \int_{\frac{\pi}{2 L}}^{\infty} e^{-m^{2} s} \ln (2|\sin L s|) d s
\end{array}
$$

By using the formula [18]

$$
\int_{0}^{\infty} e^{-q x} \ln (2|\sin a x|) d x=-q \sum_{k=1}^{\infty} \frac{1}{k\left(q^{2}+4 k^{2} a^{2}\right)}, \operatorname{Re} q>0
$$

and the fact that the integral $M \int_{1 / \Lambda^{2}}^{\frac{\pi}{2 L}} \ln (2|\sin L s|) d s$ is finite, we conclude that the integral $m^{2} M \int_{\frac{\pi}{2 L}}^{\infty} e^{-m^{2} s} \ln (2|\sin L s|) d s$ tends to zero on the critical line (where $m^{2} \rightarrow$ $0)$. Thus, v.p. $\int_{\frac{\pi}{2 L}}^{\infty} d s e^{-s m^{2}} M L \cot (L s)=-M \ln 2$. Therefore, in view of (9), we have

$$
v \cdot p \cdot \int_{\frac{\pi}{2 L}}^{\infty} d s e^{-s m^{2}}(M \operatorname{coth}(M s)-1 / s) L \cot (L s) \leq-M \ln 2
$$

It remains to estimate from below the integral $\int_{1 / \Lambda^{2}}^{\frac{\pi}{2 L}} d s(M \operatorname{coth}(M s)-1 / s) L \cot (L s)$. We have $\int_{1 / \Lambda^{2}}^{\frac{\pi}{2 L}} d s(M \operatorname{coth}(M s)-1 / s) L \cot (L s) \leq \int_{1 / \Lambda^{2}}^{\frac{\pi}{2 L}}(M \cot (M s)-1 / s) \frac{d s}{s}$ (because $\cot \mathrm{x} \leq 1 / x$ for $\mathrm{x}$ in the interval from 0 to $\left.\frac{\pi}{2}\right)$. If $M \gg L$, then we use the estimate $\operatorname{coth} \mathrm{x} \leq 1 / \mathrm{x}+1$ because $\operatorname{coth}(M s)$ is approximately 1 near the upper limit of integration. Therefore, in this case

$$
\int_{1 / \Lambda^{2}}^{\frac{\pi}{2 L}} d s(M \operatorname{coth}(M s)-1 / s) L \cot (L s) \leq M \ln \frac{\pi \Lambda^{2}}{2 L} .
$$

If $M \ll L$, then $\operatorname{coth}(M s) \ll 1$ in the interval of integration. By using the estimate $\operatorname{coth} \mathrm{x} \leq \frac{1}{x}+x / 3\left(\frac{1}{x}\right.$ and $\mathrm{x} / 3$ are simply two first terms of the Teylor expansion of cothx), we obtain

$$
\int_{1 / \Lambda^{2}}^{\frac{\pi}{2 L}} d s(M \operatorname{coth}(M s)-1 / s) L \cot (L s) \leq \frac{M^{2}}{3}\left(\frac{\pi}{2 L}-1 / \Lambda^{2}\right)
$$


We now analyse the obtained results. We assume in what follows that $\left|f_{1}\right| \gg\left|f_{2}\right|$. In the magnetic-type case $\left(f_{1}>0\right)$, we have $L \approx|e|\left(\frac{f_{2}^{2}}{f_{1}}\right)^{1 / 2}$ and $M \approx|e| f_{1}^{1 / 2}$. By using (8), (12), and (13), we obtain the following estimate from below for the critical coupling constant in the magnetic-type case:

$$
g_{c r} \geq \frac{1}{1-\frac{L \pi}{2 \Lambda^{2}}+\frac{M}{\Lambda^{2}} \ln \frac{\Lambda^{2}}{2 L}-\frac{M}{\Lambda^{2}} \ln 2} \approx \frac{1}{1+|e| \frac{f_{1}^{1 / 2}}{\Lambda^{2}} \ln \frac{\Lambda^{2} f_{1}^{1 / 2}}{4\left|e f_{2}\right|}},
$$

where $g_{c r}$ is the dimensionless critical coupling constant $g_{c r}=\frac{4 \pi^{2} G \Lambda^{2}}{N}$. It directly follows from (15) that the presence of electric field parallel to magnetic field is very important. Indeed, if $f_{2} \neq 0$, then $g_{c r}$ is no longer equal to zero (even if the magnetic field is very strong $|\vec{B}| \sim \Lambda^{2}$ ) in contrast to the case of purely magnetic field where $g_{c r}=0$. If $f_{2}$ increases, $g_{c r}$ is also increases. If $f_{2} \rightarrow 0$, then $g_{c r} \rightarrow 0$, i.e. we recover the result obtained by Gusynin, Miransky, and Shovkovy [10]. In the electric-type case $\left(f_{1}<0\right)$ we have $L \approx|e|\left|f_{1}\right|^{1 / 2}$ and $M \approx|e|\left(\frac{f_{2}^{2}}{\left|f_{1}\right|}\right)^{1 / 2}$. By using (8), (12), and (14), we obtain

$$
g_{c r} \geq \frac{1}{1-\frac{L \pi}{2 \Lambda^{2}}+\frac{M^{2}}{3 \Lambda^{2}}\left(\frac{\pi}{2 L}-1 / \Lambda^{2}\right)-\frac{M}{\Lambda^{2}} \ln 2} \approx \frac{1}{1-\frac{\pi|e|\left|f_{1}\right|^{1 / 2}}{2 \Lambda^{2}}-\frac{|e|}{\Lambda^{2}}\left(\frac{f_{2}^{2}}{\left|f_{1}\right|}\right)^{1 / 2} \ln 2} .
$$

It follows from (16) that in this case $g_{c r}$ for $f_{2} \neq 0$ is more than $g_{c r}=\frac{1}{1-\frac{\pi|e|\left|f_{1}\right|^{1 / 2}}{2 \Lambda^{2}}}[13]$ in the case $f_{2}=0$. As $f_{2}$ goes to zero, our estimate coincides with the result obtained by Klevansky and Lemmer [13] in the electric-type case. Thus, we conclude from the obtained estimates that the second invariant of electromagnetic field inhibits $D \chi S B$. In magnetic-type case it looks rather natural (indeed, if $f_{2} \neq 0$, then it means that $\vec{E} \neq 0$ and we know that electric field inhibits $D \chi S B$ ). However, in the electric-type case it appears unlikely. Indeed, let first $\vec{E} \neq 0, \vec{B}=0$ (therefore, $\left.f_{2}=0\right)$. It is natural to assume that $g_{c r}$ should decrease in the case $f_{2} \neq 0$ because if $f_{2} \neq 0$ it means that $\vec{B} \neq 0$ and we know that magnetic field assists $D \chi S B$. We can understand the cause of growth of $g_{c r}$ with increasing of $\vec{E} \cdot \vec{B}$ as follows. Since we study the dependence on the second invariant, we keep the first invariant $\vec{B}^{2}-\vec{E}^{2}$ unchanged. Without loss of generality we can assume that $\vec{E} \| \vec{B}$ (if not, one can 
perform an appropriate Lorentz transformation). If we increase $f_{2}$, then in order to keep the first invariant unchanged we have to increase both $\vec{B}$ and $\vec{E}$. Therefore, there is a competition between increasing of $\vec{B}$ and increasing of $\vec{E}$. It turned out that qualitatively increasing of $\vec{E}$ is more significant for $g_{c r}$ than increasing of $\vec{B}$ for any $f_{1}$, therefore, $g_{c r}$ always grows with increasing of $f_{2}$.

We found a rather rough analytic estimate from below for the critical coupling constant. To obtain a more accurate dependence of the critical coupling constant on $f_{2}$, we numerically calculate the integral in (7). A typical dependence of $g_{c r}$ on $f_{2}$ in the electric-type case is shown in Fig.1 (this figure corresponds to $\frac{f_{1}}{\Lambda^{4}}=-10^{-4}$ ) and in the magnetic-type case in Fig.2 (where $\frac{f_{1}}{\Lambda^{4}}=10^{-4}$ ).

We see from these figures that the critical coupling constant increases with increasing of $f_{2}$ for any value of $f_{1}$. In the electric-type case $g_{c r}$ is always more that 1. In the magnetic-type case $g_{c r}$ abruptly drops to zero as $f_{2}$ tends to zero. We also numerically calculated $g_{c r}$ in the case where the first invariant is zero $f_{1}=0$ $(|\vec{E}|=|\vec{B}|)$ and obtained a dependence which is similar to the electric-type case, i.e. $g_{c r}$ increases with increasing of $f_{2}$. Thus, the numerical analysis of the gap equation confirms that the second invariant of electromagnetic field inhibits $D \chi S B$.

We now consider the case of $2+1$ dimensions. We use the reducible 4 -dimensional representation of the Dirac algebra for fermion field in order that the model possess a chiral symmetry (in fact, there is two chiral symmetries with $\gamma_{5}$ and $\gamma_{3}$ matrices, for more details see [19]) and we do not study parity breaking. Thus, we have the following gap equation for parity conserving mass:

$$
m=2 i G \operatorname{tr}\left(S_{A}^{(m)}(x, x)\right)
$$

where $S_{A}^{(m)}$ is the fermion propagator in an external constant electromagnetic field which has the following form in the Fock-Schwinger proper time formalism:

$$
S_{A}^{(m)}\left(x, x^{\prime}\right)=\left(i \partial_{\mu} \gamma^{\mu}-e A_{\mu} \gamma^{\mu}-m\right)(-i) \int_{-\infty}^{0} d \tau U_{A}\left(x, x^{\prime} ; \tau\right)
$$


where $\epsilon \rightarrow 0$ and

$$
\begin{aligned}
U_{A}\left(x, x^{\prime} ; \tau\right)= & <x\left|\frac{1}{(i \partial-e A)^{2}-\frac{e}{2} \sigma^{\mu \nu} F_{\mu \nu}-m^{2}}\right| x^{\prime}> \\
= & \frac{e^{-i \frac{\pi}{4}}}{8 \pi^{3 / 2}|\tau|^{3 / 2}} \exp \left[-i e \int_{x^{\prime}}^{x} d \xi A(\xi)+\right. \\
& \frac{i}{4}\left(x-x^{\prime}\right) e F \operatorname{coth}(e F \tau)\left(x-x^{\prime}\right)- \\
& \left.\frac{1}{2} \operatorname{tr}\left[\ln \left(\frac{\sinh e F \tau}{e F \tau}\right)\right]+\frac{i e}{2} \sigma F \tau+i m^{2} \tau\right] .
\end{aligned}
$$

By taking trace over the Dirac indices in (13), we obtain

$$
m=\frac{m G}{\pi^{3 / 2}} e^{-i \pi / 4} \int_{-\infty}^{0} \frac{d \tau}{|\tau|^{3 / 2}}(e X \tau) \cot (e X \tau)
$$

where $X=\sqrt{\frac{1}{2} F_{\mu \nu} F^{\mu \nu}}=\sqrt{B^{2}-\vec{E}^{2}}$ (note that magnetic field is a pseudoscalar in $2+1$ dimensions, not axial-vector).

As well known, in $2+1$ dimensions there is only one Lorentz invariant $\frac{1}{2} F_{\mu \nu} F^{\mu \nu}$ of electromagnetic field. Indeed, we can see from (20) that the gap equation depends on electromagnetic fields only through this Lorentz invariant. Consequently, an arbitrary combination of constant fields can be reduced to cases either purely magnetic or purely electric field. The case of constant magnetic field in the NambuJona-Lasinio model in $2+1$ dimensions was considered in [10]. Therefore, we study here only the case of constant electric field.

In the case of external electric field the gap equation takes the form

$$
\begin{aligned}
m= & \frac{m G}{\pi^{3 / 2}} e^{-i \pi / 4} \int_{\frac{1}{\Lambda^{2}}}^{\infty} \frac{d s}{s^{3 / 2}} e^{-s m^{2}}+ \\
& \frac{m G}{\pi^{3 / 2}} e^{-i \pi / 4} \int_{-\infty}^{0} \frac{d \tau}{|\tau|^{3 / 2}} e^{i \tau\left(m^{2}-i \epsilon\right)}[(e E \tau) \operatorname{coth}(e E \tau)-1]
\end{aligned}
$$

where we explicitly wrote down the term which corresponds to the gap equation without external electric field and $E=|\vec{E}|$. Further, by using the fact that $\tau \operatorname{coth} \tau-1=\tau\left(1+\frac{2}{e^{2 \tau}-1}-\frac{1}{\tau}\right)$ and performing the change of variable $x=\frac{\tau}{2}$, for $\operatorname{Re}\left[e^{-i \pi / 4} \int_{-\infty}^{0} \frac{d \tau}{|\tau|^{3 / 2}} e^{i \tau\left(m^{2}-i \epsilon\right)}(e E \tau) \operatorname{coth}(e E \tau)\right]$ we get

$$
\operatorname{Re}\left[I_{E}\left(\mu_{E}^{2}, \frac{1}{2}\right)\right]-\operatorname{Im}\left[I_{E}\left(\mu_{E}^{2}, \frac{1}{2}\right)\right],
$$


where $I_{E}\left(\mu_{E}^{2}, \frac{1}{2}\right)=\int_{0}^{\infty} x^{a-1} e^{i \frac{\mu_{E}^{2}}{2} x}\left(\frac{1}{e^{x}-1}-\frac{1}{x}\right), \mu_{E}^{2}=\frac{m_{E}^{2}}{e E}$ and we also used the equality

$$
\frac{1}{2} \int_{0}^{\infty} \frac{d x}{x^{1 / 2}}\left(\cos \left(\frac{\mu_{E}^{2}}{2} x\right)-\sin \left(\frac{\mu_{E}^{2}}{2} x\right)\right)=0
$$

$I_{E}\left(\mu_{E}^{2}, a\right)$ is an analytic function of a in the region $0<\operatorname{Re}(a)<2$.

For $1<\operatorname{Re}(a)<2$, by representing $I_{E}\left(\mu_{E}^{2}\right)$ as a sum of two integrals, we have

$$
I_{E}\left(\mu_{E}^{2}, a\right)=-\left(\frac{2}{\mu_{E}^{2}}\right)^{a-1} \Gamma(a-1) e^{i \frac{a-1}{2} \pi}+\Gamma(a) \zeta\left(a, 1-i \frac{\mu_{E}^{2}}{2}\right) .
$$

Performing an analytic continuation of $(24)$ to the region $0<\operatorname{Re}(a)<2$, we get

$$
I_{E}\left(\mu_{E}^{2}, \frac{1}{2}\right)=\sqrt{\pi}\left((1-i) \mu_{E}+\zeta\left(\frac{1}{2}, 1-i \frac{\mu_{E}^{2}}{2}\right)\right) .
$$

Consequently, the gap equation takes the form

$$
\frac{\pi^{3 / 2}}{2 G \Lambda}=1+\frac{\sqrt{\pi}(e E)^{1 / 2}}{2 \Lambda}\left(\operatorname{Re}\left[\zeta\left(\frac{1}{2}, 1-i \frac{\mu_{E}^{2}}{2}\right)\right]-\operatorname{Im}\left[\zeta\left(\frac{1}{2}, 1-i \frac{\mu_{E}^{2}}{2}\right)\right]\right)+O\left(\frac{m^{2}}{\Lambda^{2}}\right)
$$

From (26), we obtain the following value for the critical coupling constant:

$$
g_{c r}(E)=\frac{\pi^{3 / 2}}{2 \Lambda} \frac{1}{1-a^{2} \zeta\left(\frac{1}{2}\right)\left(\frac{e E}{\Lambda^{2}}\right)^{1 / 2}}=\frac{g_{c r}(0)}{1-a^{2}\left(\frac{e E}{\Lambda^{2}}\right)^{1 / 2}}
$$

where $g_{c r}=\frac{2 G \Lambda^{2}}{\pi^{3 / 2}}$ is the dimensionless coupling constant in $2+1$ dimension and $a^{2}=-\frac{\sqrt{\pi}}{2} \zeta\left(\frac{1}{2}\right) \approx 1.29$.

Thus, the presence of external constant electric field increases the value of critical coupling constant. Note that the same is true in $3+1$ dimensions (see [13]). It is not difficult to show that in the vicinity of critical point

$$
m^{2} \approx C^{2}\left(E_{c r}-E\right)^{1 / 2}
$$

where $C^{2}=-2 \frac{\zeta(1 / 2)}{\zeta(3 / 2)} \approx 1.12$.

Thus, this phase transition is a phase transition of the second order.

Since we have only one Lorentz invariant of electromagnetic field $X^{2}=\frac{1}{2} F_{\mu \nu} F_{\mu \nu}=$ $B^{2}-\vec{E}^{2}$ in $2+1$ dimensions, by using an appropriate frame, the general case of non-zero constant electromagnetic field can be reduced to the cases of purely electric or purely magnetic fields. 
The authors are grateful to Prof. V.P. Gusynin for many fruitful discussions and remarks, Prof. V.A. Miransky for valuable comments, and Dr. I.A. Shovkovy for the help in drawing figures. The work of E.V.G. was supported in part through grant INTAS-93-2058-EXT "East-West network in constrained dynamical systems" and by the Foundation of Fundamental Research of the Ministry of Science of the Ukraine through grant No. 2.5.1/003. The work of A.Yu.B. was supported in part by the International Soros Science Education Program (ISSEP) through grant No. GSU062075.

\section{References}

[1] T. Maskawa and H. Nakajima, Progr. Theor. Phys. 52, No. 4 (1974) 1326.

[2] R. Fukuda and T. Kugo, Nucl Phys. B117, No. 1 (1976) 250.

[3] P.I. Fomin and V.A. Miransky, Phys. Lett. B64, No. 2 (1976) 166;

P.I. Fomin, V.P. Gusynin, V.A. Miransky, and Yu.A. Sitenko, Riv. Nuovo Cim. 6, No. 5 (1983) 1 .

[4] V.A. Miransky, Nuovo Cim. A90 (1985) 149.

[5] C.N. Leung, S.T. Love, and W.A. Bardeen, Nucl. Phys. B273, No. 3 (1986) 649.

[6] A. Kocic, E. Daggotto, and J.B. Kogut, Phys. Lett. B213, No. 1 (1988) 56.

[7] T. Akiba and T. Yanagida, Phys. Lett. B169, No. 4 (1986) 432;

K. Yamawaki, M. Bando, and K. Matumoto, Phys. Rev. Lett. 56 (1986) 1335;

B. Holdom, Phys. Lett. B150 (1985) 301;

T. Appelquist, D. Karabali, and L.C.R. Wijewardhana, Phys. Rev. Lett. 57 (1986) 957.

[8] D. G. Caldi and A. Chodos, Phys. Rev. D36 (1987) 2876. 
[9] Y.J. Ng and Y. Kikuchi, Phys. Rev. D36 (1987) 2880.

[10] V.P. Gusynin, V.A. Miransky, and I.A. Shovkovy, Phys. Rev. Lett. 73 (1994) 3499 ;

V.P. Gusynin, V.A. Miransky, and I.A. Shovkovy, Phys. Rev. D52 (1995) 4718.

[11] V.P. Gusynin, V.A. Miransky, and I.A. Shovkovy, Phys. Lett. B349 (1995) 477.

[12] Y. Nambu and G. Jona-Lasinio, Phys. Rev. 122 (1961) 345.

[13] S.P. Klevansky and R.H. Lemmer, Phys. Rev. D39 (1989) 3478.

[14] S.P. Klevansky, Rev. Mod. Phys. 64 (1992) 649;

I.V. Krive and S.A. Naftulin, Phys. Rev. D46 (1992) 2737;

K.G. Klimenko, Z. Phys. C54 (1992) 323.

[15] V.P. Gusynin, V.A. Miransky, and I.A. Shovkovy, Phys. Rev. D52 (1995) 4747;

C.N. Leung, Y.J. Ng, and A.W. Ackley, Phys. Rev. D54 (1996) 4181;

D.K. Hong, W. Kim, and S.-J. Sin, Phys. Rev. D54 (1996) 7879.

[16] V.A. Fock, Sov. Phys. 12 (1937) 404.

[17] J. Schwinger, Phys. Rev. 82 (1951) 664.

[18] A.P. Prudnikov, Yu.A. Brychkov, O.I. Marichev, Integrals and series. Elementary functions (Nauka, Moscow, 1981, in Russian).

[19] T. Appelquist, M. Bowick, D. Karabali, and L.C.R. Wijewardhana, Phys. Rev. D33 (1986) 3774 . 


\section{Figure captions}

Figure 1: The dependence of the critical coupling constant on the second invariant in the magnetic-type case.

Figure 2: The dependence of the critical coupling constant on the second invariant in the electric-type case. 


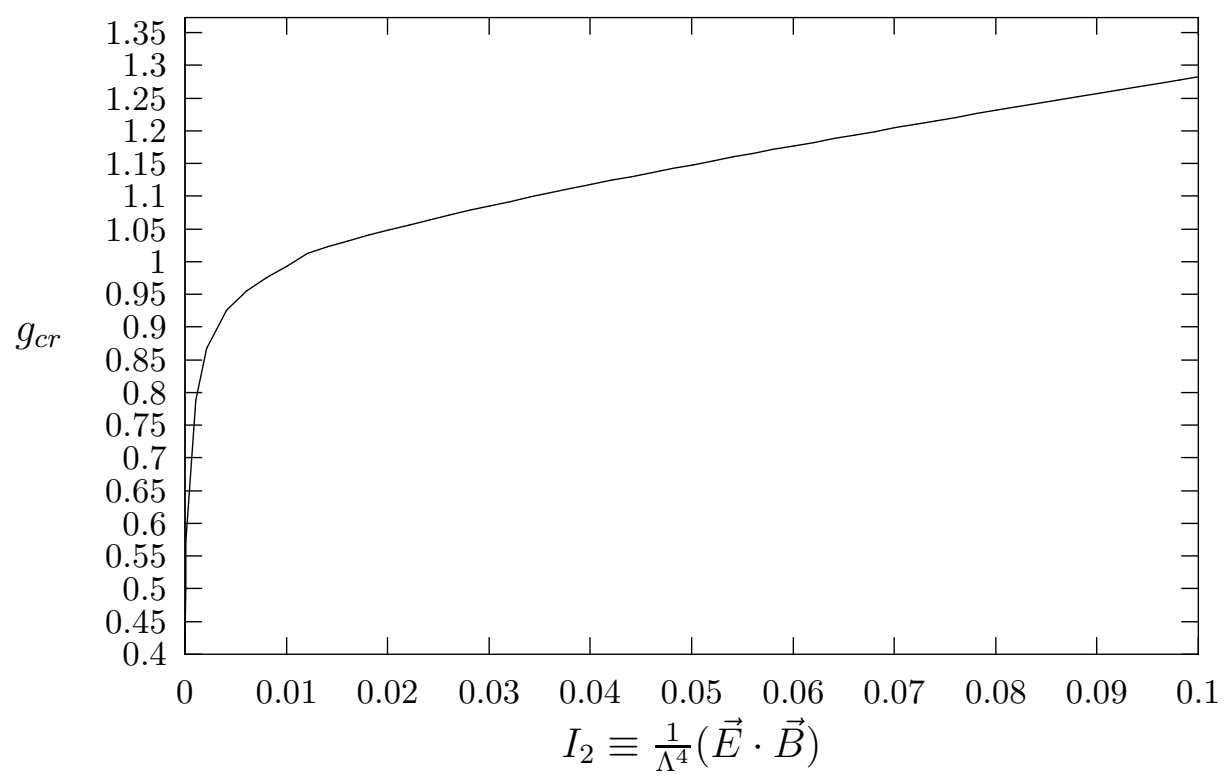

Fig.1

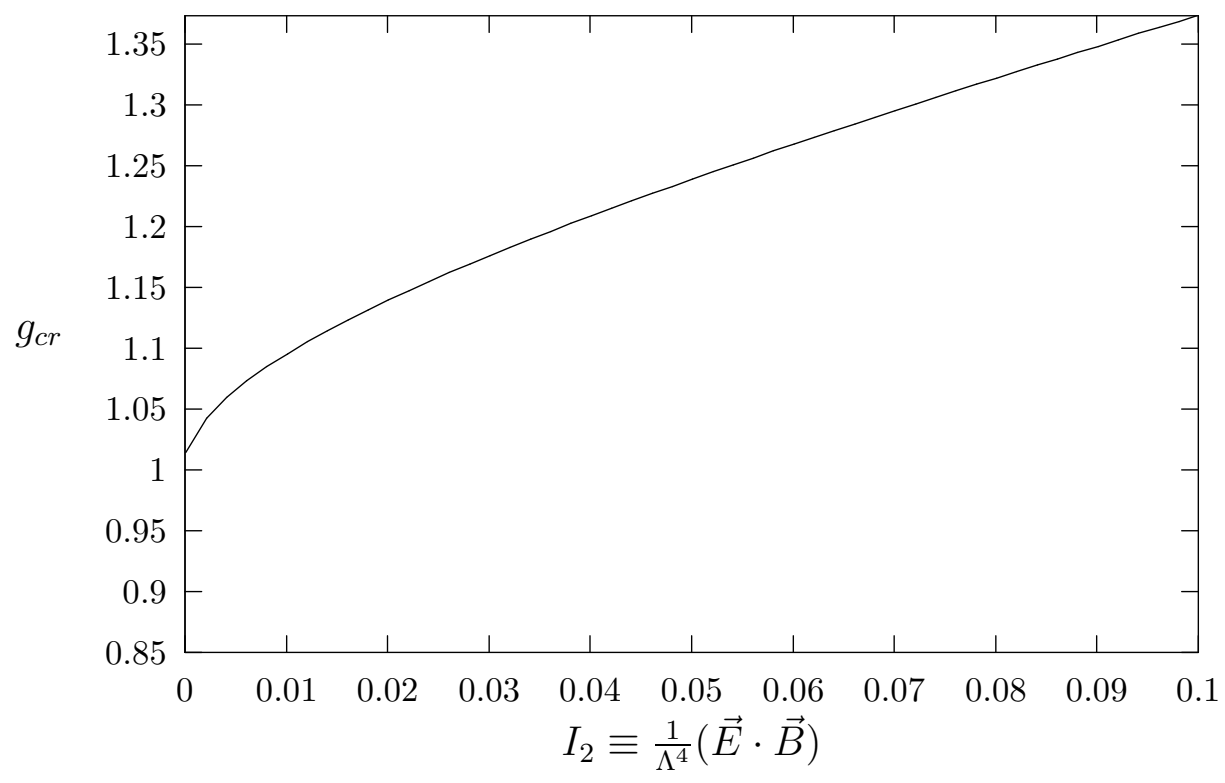

Fig.2 